\title{
Ultrasonido endobronquial para el diagnóstico de tumores pulmonares de localización central: Revisión sistemática y metaanálisis
}

\author{
Jolanda C. Kuijvenhoven ${ }^{\mathrm{a}, \mathrm{b}}$ Fausto Leoncini ${ }^{\mathrm{a}, \mathrm{c}}$ Laurence C. Crombag ${ }^{\mathrm{a}}$ René Spijker ${ }^{\mathrm{d}}$ \\ Peter I. Bonta ${ }^{a}$ Daniël A. Korevaar ${ }^{\mathrm{e}}$ Jouke T. Annema ${ }^{\mathrm{a}}$ \\ a Departamento de Medicina Respiratoria, Centro Médico Académico, Universidad de Ámsterdam, Ámsterdam, Países Bajos; \\ ${ }^{b}$ Departamento de Medicina Respiratoria, Medisch Centrum Leeuwarden, Leeuwarden, Países Bajos; \\ 'Sección de Medicina Respiratoria, Hospital Universitario Careggi, Florencia, Italia; \\ dBiblioteca Médica, Centro Médico Académico, Universidad de Ámsterdam, Ámsterdam, Países Bajos; \\ eDepartamento de Epidemiología Clínica, Bioestadística y Bioinformática, Centro Médico Académico, Universidad de Ámsterdam, \\ Ámsterdam, Países Bajos
}

\section{Palabras clave}

Cáncer pulmonar · Diagnóstico · Ultrasonido endobronquial

Tumor

\section{Resumen}

Introducción: Es difícil obtener un diagnóstico tisular de tumores pulmonares de localización central en pacientes que no presentan anormalidades endobronquiales, y por tanto es un problema diagnóstico de consideración.

Objetivo: El objetivo de este estudio fue evaluar el desempeño del ultrasonido endobronquial lineal con biopsia transbronquial por aspiración con aguja (USEB-BTBA) para el diagnóstico de tumores pulmonares de localización central.

Métodos: Realizamos una revisión sistemática (PROSPERO, CRD42017080968) y buscamos estudios que evaluaran el rendimiento y/o la sensibilidad de USEB-BTBA para el diagnóstico de tumores pulmonares de localización central, en MEDLINE, Embase, BIOSIS Previews y Web of Science hasta el 18 de noviembre de 2018 Evaluamos la calidad del estudio mediante QUADAS-2 y efectuamos un metaanálisis de efectos aleatorios.

Resultados: En total se identificaron 5,657 manuscritos; de éstos, se consideraron 14 para el estudio, que incluyeron a 1,175 pacientes sometidos a USEB-BTBA para el diagnóstico de un tumor intrapulmonar. Todos los estudios mostraron un alto riesgo de sesgo o problemas de aplicabilidad, especialmente en la selección de los pacientes. El rendimiento promedio de USEB-BTBA para diagnosticar tumores pulmonares de localización central fue 0.89 (IC 95\% 0.84-0.92), y la sensibilidad promedio para diagnosticar tumores malignos fue 0.91 (IC 95\% 0.88-0.94). Entre los estudios que publican esta información ocurrieron complicaciones relacionadas con el USEB en 5.4\% de los pacientes (42/721).

Conclusión: El USEB-BTBA tiene alto rendimiento y sensibilidad para diagnosticar tumores pulmonares de localización central, y es segura en pacientes seleccionados. Se recomienda emprender estudios prospectivos para evaluar el uso rutinario de este procedimiento en el diagnóstico de tumores intrapulmonares.

C 2019 El (los) autor(es). Publicado por S. Karger AG, Basilea

\section{Introducción}

El cáncer pulmonar es la causa principal de mortalidad relacionada con neoplasias en el mundo [1]. Si se sospecha la presencia de cáncer pulmonar, un diagnóstico tisular es necesario para establecer un diagnóstico definitivo. En pacientes con tumores pulmonares de localización central en quienes se sospecha cáncer pulmonar, los lineamientos clínicos actuales recomiendan una information@karger.com

www.karger.com/kxn

Karger ${ }^{\prime \prime}=$ $\begin{array}{ll}\text { (C) } 2019 \mathrm{El} \text { (los) autor(es) } & \text { Karger } \\ \text { Publicado por S. Karger AG, Basilea } & \text { Open caccess }\end{array}$

This article is licensed under the Creative Commons Attribution 4.0 International License (CC BY) (http://www.karger.com/Services/OpenAccessLicense). Usage, derivative works and distribution are permitted provided that proper credit is given to the author and the original publisher.
Jouke T. Annema, MD, PhD

Departamento de Medicina Respiratoria, Centro Médico Académico

Universidad de Ámsterdam

Meibergdreef 9, 1105 AZ Ámsterdam, Países Bajos

j.t.annema@amc.uva.nl 
broncoscopía flexible convencional con biopsia o una biopsia transbronquial por aspiración con aguja (BTBA) para obtener un diagnóstico [2]. Sin embargo, la broncoscopía no arroja resultados informativos en una proporción considerable de pacientes, especialmente en ausencia de anormalidades endobronquiales [3]. Puede utilizarse la biopsia transtorácica por aspiración con aguja fina guiada por tomografía computarizada (TC) para obtener un diagnóstico, pero en los tumores pulmonares de localización central esta técnica presenta un alto riesgo de complicaciones, como neumotórax y hemorragia [3]. Por otra parte, con frecuencia dichos tumores son inaccesibles en un abordaje transtorácico, y el rendimiento diagnóstico es menor que para tumores pulmonares periféricos $[4,5]$.

Los lineamientos actuales de estadificación recomiendan el ultrasonido endobronquial (USEB) y esofágico (USE-[B]) como las técnicas de elección para la estadificación tisular de nódulos en el mediastino en el cáncer pulmonar no-microcítico [6-8]. Para pacientes en quienes las imágenes por TC muestran un tumor pulmonar de localización central adyacente a las vías respiratorias mayores, se sugiere utilizar el ultrasonido endobronquial con biopsia transbronquial por aspiración con aguja (USEB-BTBA) para establecer un diagnóstico, luego de una broncoscopía convencional sin resultados informativos $[6,9]$.

Aunque el uso de la técnica de USEB se ha extendido para la estadificación de nódulos en el mediastino en el cáncer pulmonar, su utilidad para obtener una muestra adecuada de tejido directamente de tumores intrapulmonares ha recibido mucho menos atención. Si es lo suficientemente accesible y exacta, el diagnóstico de tumores pulmonares mediante USEB podría ofrecer grandes ventajas logísticas, puesto que pueden estadificarse los nódulos en el mediastino y el tumor en una misma sesión [6, 10].

Se han publicado varios reportes sobre el papel del USEB-BTBA en el diagnóstico de tumores pulmonares de localización central, pero su factibilidad, rendimiento, sensibilidad y seguridad no están bien establecidas [6]. Por tanto, emprendimos una revisión sistemática y un metaanálisis con el objetivo de obtener estimaciones sumarias del rendimiento y la sensibilidad del USEB-BTBA para diagnosticar tumores de localización central en pacientes bajo sospecha de cáncer pulmonar.

\section{Material y métodos}

El protocolo de esta revisión sistemática se registró prospectivamente en PROSPERO bajo el número de registro CRD42017080968. Esta revisión se publica siguiendo los lineamientos PRISMA-DTA [11].

\section{Criterios de elegibilidad}

Se incluyeron estudios que evaluaron el rendimiento y/o la sensibilidad del USEB-BTBA para diagnosticar tumores pulmonares de localización central adyacentes o cercanos a las vías respiratorias mayores, con el propósito de obtener una muestra de tejido de la lesión sospechosa. Existen varias definiciones de tumor pulmonar de localización central, y seguimos las que ofrecieron los autores de los estudios primarios. Los estudios se consideraron elegibles para el análisis aun si los pacientes fueron seleccionados con base en los resultados de pruebas anteriores. También se incluyeron estudios cuyo objetivo era obtener un diagnóstico tisular de tumores pulmonares de localización central que invadieron el mediastino o los vasos centrales. Sin embargo, excluimos estudios enfocados en el diagnóstico de tumores mediastinales, estudios cuyo objetivo era diagnosticar el cáncer pulmonar mediante muestras de los nódulos del mediastino, lesiones hepáticas o lesiones en la glándula adrenal izquierda, y estudios que emplearon una sonda de USEB radial en vez de una lineal, así como estudios que incluyeron $<10$ pacientes con tumores pulmonares de localización central.

\section{Estrategia y selección de la búsqueda en la literatura}

Buscamos estudios elegibles en MEDLINE (Ovid), Embase (Ovid), BIOSIS Previews (Ovid), y Web of Science. Un especialista en información médica (R.S.) desarrolló las búsquedas. No se aplicaron restricciones con respecto a la fecha o al lenguaje. La estrategia completa de búsqueda se ofrece en línea en el Apéndice suplementario (Tabla S1 supl.; para ver el material supl. original en línea, vea www. karger.com/doi/10.1159/000500363). La búsqueda final se realizó el 18 de noviembre de 2018. Verificamos las listas de referencias de todos los artículos incluidos para buscar estudios adicionales.

Dos autores (J.C.K. y L.C.C.) revisaron independientemente los títulos y resúmenes de todos los resultados de la búsqueda para verificar la elegibilidad. Si un artículo se consideró potencialmente elegible, ambos autores examinaron independientemente el artículo completo, para evaluar su inclusión. Cualquier desacuerdo se resolvió tras discutirlo con un tercer autor (J.T.A.).

\section{Extracción y síntesis de datos}

Dos autores (J.C.K. y F.L.) extrajeron los datos de los estudios incluidos. Extrajimos el primer autor, el año y la revista de publicación, y el país donde se reclutaron los pacientes. También determinamos si los pacientes se habían sometido a pruebas anteriores para obtener un diagnóstico del tumor de localización central con base en una biopsia. Asimismo, extrajimos detalles sobre la edad y el género, la disponibilidad de una evaluación citológica rápida in situ (ROSE), el tipo de aguja, el número de pases de aguja efectuados, la duración del procedimiento, el tamaño del tumor, el número de pacientes con anormalidades endobronquiales, el estándar de referencia para el diagnóstico y cualquier complicación inducida por el USEB-BTBA.

Adicionalmente, extrajimos el número total de pacientes sometidos a USEB-BTBA con el fin de diagnosticar un tumor pulmonar de localización central, el número de pacientes en quienes se obtuvo una muestra tisular adecuada por USEB-BTBA, el número de pacientes en quienes el USEB-BTBA permitió un diagnóstico correcto (maligno o no) con una biopsia como evidencia, el número de pacientes en quienes el USEB-BTBA permitió diagnosticar un tumor maligno, y el número de pacientes para quienes el tumor intrapulmonar estudiado resultó maligno de acuerdo con el estándar de referencia. 
Fig. 1. Diagrama de flujo del proceso de selección de los estudios incluidos. USEB, ultrasonido endobronquial.

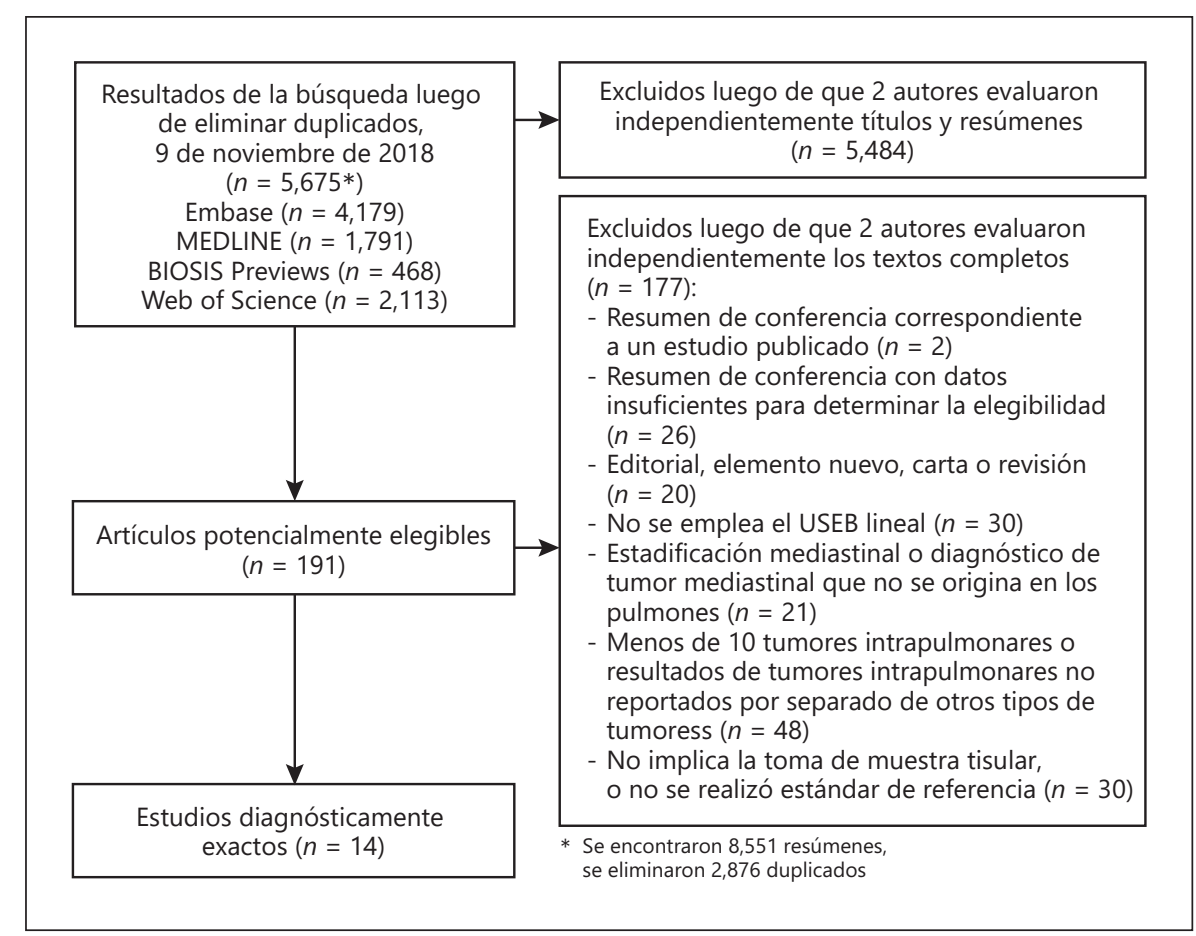

Se consideró que el USEB-BTBA alcanzó un diagnóstico inadecuado si se requirieron procedimientos adicionales para obtener un diagnóstico correcto (p. ej., porque el tumor no pudo visualizarse o porque fue imposible tomar una muestra de él mediante USEB-BTBA) o si el estándar de referencia llevó a un diagnóstico diferente. Se consideró que el USEB-BTBA alcanzó un diagnóstico correcto si el estándar de referencia llegó al mismo diagnóstico, o si las muestras de tejido obtenidas mediante USEB-BTBA contuvieron células malignas, puesto que el estándar de referencia rara vez se efectúa en tales casos.

Evaluación del riesgo de sesgo y problemas de aplicabilidad Dos autores (J.C.K. y F.L.) evaluaron independientemente la calidad del estudio utilizando la herramienta QUADAS-2 [12]. Los desacuerdos se resolvieron por consenso, y en casos difíciles, otros dos autores (L.C.C. y D.A.K.) tomaron la decisión final. En un diseño de estudio con alto riesgo de sesgo o problemas de aplicabilidad se observó: (1) la inclusión retrospectiva (no-consecutiva) de pacientes; (2) la exclusión de pacientes en quienes el tumor intrapulmonar no pudo visualizarse por USEB; (3) un diseño de casos y controles; (4) la exclusión de pacientes que no correspondían a la pregunta de revisión; (5) endoscopistas que no desconocían el diagnóstico final mientras realizaban el USEB; (6) un estándar de referencia subóptimo para pacientes con una USEB-BTBA con resultados no informativos o sin malignidad (p. ej., seguimiento clínico en vez de verificación quirúrgico-patológica); (7) verificación parcial o (8) diferencial de pacientes con una USEB-BTBA sin información diagnóstica o sin malignidad; (9) la exclusión de pacientes para quienes no existían resultados del estándar de referencia.

\section{Resultados primarios}

Los resultados primarios de esta revisión fueron: (1) el rendimiento del USEB-BTBA para el diagnóstico de tumores pulmonares de localización central y (2) la sensibilidad del USEB-BTBA para el diagnóstico de tumores pulmonares malignos de localización central.

El rendimiento se definió como el número de pacientes para quienes el USEB-BTBA permitió un diagnóstico tisular correcto, con respecto al número total de pacientes en quienes se practicó el USEB con el fin de diagnosticar un tumor pulmonar de localización central.

La sensibilidad se definió como el número de pacientes para quienes USEB-BTBA permitió el diagnóstico tisular correcto de un tumor maligno, con respecto al número total de pacientes en quienes el tumor pulmonar de localización central bajo estudio resultó ser maligno.

\section{Análisis}

Calculamos estimaciones del rendimiento y la sensibilidad de los estudios incluidos con un IC 95\%, utilizando la aproximación normal. Posteriormente hicimos un metaanálisis de efectos aleatorios siguiendo a DerSimonian-Laird [13]. Los datos se analizaron en el paquete «meta» en $\mathrm{R}$, versión 3.0.

\section{Resultados}

\section{Selección y características de los estudios}

Las búsquedas identificaron 5,675 resultados. Después de examinar los títulos y resúmenes, se conservaron 191 artículos potencialmente elegibles, y de ellos, 14 estudios se incluyeron en el análisis final [9, 14-26]. De éstos, 3 fueron resúmenes de conferencia. 
Tabla 1. Definiciones de las lesiones pulmonares evaluadas

\begin{tabular}{|c|c|}
\hline Estudio [ref], año & Los tumores intrapulmonares evaluados se definieron como \\
\hline Nakajima et al. [9], 2008 & $\begin{array}{l}\text { Masas pulmonares cuyo bronquio de drenaje es difícil de alcanzar, tales como el cáncer de pulmón de tipo } \\
\text { mediastinal adyacente a la tráquea, lesiones adyacentes al bronquio principal o al bronquio segmentario }\end{array}$ \\
\hline Tournoy et al. [14], 2009 & $\begin{array}{l}\text { Las lesiones pulmonares de localización central se definieron como una masa intrapulmonar con el margen medial } \\
\text { ubicado en el tercio interno del hemitórax, con base en imágenes de TC exploratoria }\end{array}$ \\
\hline Khan et al. [16], 2012 & Lesiones centrales del parénquima pulmonar \\
\hline Bhatti et al. [17], 2013 & Lesiones pulmonares peribronquiales de localización central \\
\hline Verma et al. [18], 2013 & $\begin{array}{l}\text { Las lesiones pulmonares de localización central se definieron como un nódulo o una masa intrapulmonar y cuya } \\
\text { ubicación es adyacente al árbol traqueobronquial, como se aprecia en una TC exploratoria del tórax }\end{array}$ \\
\hline Yang et al. [19], 2013 & Lesiones intrapulmonares parabraquiales o parabronquiales evidenciadas por TC exploratoria \\
\hline Zhao et al. [20], 2013 & Lesiones intrapulmonares localizadas en la proximidad de la vía respiratoria central \\
\hline Evison et al. [21], 2013 & Lesiones pulmonares intraparenquimatosas \\
\hline Argento et al. [22], 2016 & $\begin{array}{l}\text { Lesiones intraparenquimatosas de localización central } \\
\text { Se incluyeron lesiones completamente rodeadas por el parénquima pulmonar }\end{array}$ \\
\hline Chen et al. [23], 2017 & Lesiones pulmonares peribronquiales \\
\hline
\end{tabular}

La Figura 1 muestra los detalles de la selección de estudios, y las razones para excluir algunos estudios.

Entre los estudios incluidos se emplearon varias definiciones para los tumores pulmonares evaluados, desde «lesiones centrales en el parénquima pulmonary» [16] a «una masa intrapulmonar con el margen medial ubicado en el tercio interno del hemitórax, con base en imágenes de TC exploratoria» [14]. La Tabla 1 resume las diferentes definiciones usadas para tumores pulmonares de localización central en los estudios incluidos.

La Tabla 2 muestra las características detalladas de los 14 estudios incluidos. El primer artículo se publicó en 2008 y el último en 2018. Nueve estudios indicaron la proporción de pacientes que se sometieron previamente a una broncoscopía convencional que no ofreció información diagnóstica, y esta proporción varió de 33 a $100 \%$; no se incluyó información sobre broncoscopías previas en los otros 5 estudios. La edad media/mediana de los pacientes varió entre 56 y 69 años, y la proporción de pacientes masculinos varió de 31 a 83\%. Hubo acceso a ROSE en 6 estudios, no lo hubo en 4 estudios, y 4 estudios más no informaron sobre la disponibilidad de ROSE. Se utilizó una aguja de calibre 22 en 11 estudios, agujas de calibre 21 o 22 en 1 estudio, y el tipo de aguja empleado no se indicó en 2 estudios. Seis estudios informaron sobre el número de aspiraciones, que variaron de 2 a 6 . Tres estudios informaron la duración media/mediana del procedimiento: 21, 46 y $56 \mathrm{~min}$. El tamaño medio/mediano del tumor varió de 25 a $53 \mathrm{~mm}$. Siete estudios (542 pacientes en total) excluyeron específicamente a pacientes con anormalidades endobronquiales o no encontraron pacientes con dichas anormalidades, y 3 estudios incluyeron explícitamente pacientes con anormalidades endobronquiales (en total, se incluyeron 27 pacientes con anormalidades endobronquiales). Los 4 estudios restantes no ofrecieron comentarios sobre la presencia de pacientes con anormalidades endobronquiales.

\section{Riesgo de sesgo y problemas de aplicabilidad}

Los resultados detallados de la evaluación de la calidad de los estudios incluidos están disponibles en el Apéndice suplementario (Tabla S2 supl. original en línea). Todos los estudios tuvieron al menos un elemento que presentó un riesgo elevado de sesgo y/o problemas de aplicabilidad. La fuente de sesgo más común fue la inclusión retrospectiva de pacientes, como fue el caso en 12 de los 14 estudios incluidos. En 11 estudios no fue claro si se evitaron las exclusiones inapropiadas, como cuando se excluyeron pacientes en quienes el tumor no pudo visualizarse mediante USEB. La calidad del estándar de referencia, en ausencia de un diagnóstico específico después de aplicar el USEB, fue variable, desde la verificación quirúrgico-patológica al seguimiento clínico.

\section{Rendimiento diagnóstico y sensibilidad}

La Tabla 3 muestra las estimaciones del rendimiento y la sensibilidad de estudios individuales. El número total de pacientes incluidos en esta revisión es 1,175; el número de pacientes incluidos en los estudios individuales varió de 32 a 290. La proporción de pacientes con diagnóstico final de malignidad varió de 62 a 100\%. El diagnóstico final de malignidad incluyó cáncer pulmonar no-microcítico en 620 pacientes, carcinoma pulmonar microcítico en 126 pacientes y otro diagnóstico maligno en 61, en los 12 estudios que ofrecieron esta información. Información detallada sobre el diagnóstico final está disponible en el Apéndice suplementario en línea (Tabla S2 supl.).

El rendimiento del USEB-BTBA para el diagnóstico de lesiones intrapulmonares varió de 0.72 a 0.96 entre los estudios incluidos; un estudio no ofreció información suficiente para calcular el rendimiento. El rendimiento medio después del metaanálisis fue 0.89 (IC 95\% 0.84-0.92; Fig. 2). La sensibilidad del USEB-BTBA para diagnosticar tumores intrapulmonares malignos varió de 0.77 a
40

Kompass Neumol 2020;2:37-45

DOI: $10.1159 / 000506581$ 
Tabla 2. Características de los estudios incluidos en los que se aplicó a los pacientes un procedimiento de USEB para diagnosticar una lesión pulmonar de localización central

\begin{tabular}{|c|c|c|c|c|c|c|c|c|c|c|}
\hline $\begin{array}{l}\text { Detalles del estudio, } \\
\text { Revista } \\
\text { [ref]] }\end{array}$ & $\begin{array}{l}\text { Pruebas para obtener un } \\
\text { diagnóstico tisular antes de } \\
\text { la USEB-ATBT }\end{array}$ & $\begin{array}{l}\text { Edad, años, } \\
\text { media/ } \\
\text { mediana } \\
\text { (intervalo) }\end{array}$ & $\begin{array}{l}\text { Mascu- } \\
\text { linos, } \\
\%\end{array}$ & ROSE & $\begin{array}{l}\text { Tipo de } \\
\text { aguja }\end{array}$ & $\begin{array}{l}\text { Número de } \\
\text { aspiraciones } \\
\text { con aguja }\end{array}$ & $\begin{array}{l}\text { Duración } \\
\text { del procedi- } \\
\text { miento, } \\
\text { min }\end{array}$ & $\begin{array}{l}\text { Tamaño del } \\
\text { tumor, mm }\end{array}$ & $\begin{array}{l}\text { Estándar de referencia en } \\
\text { pacientes con una USEB-ATBT } \\
\text { sin información diagnóstica o } \\
\text { sin malignidad }\end{array}$ & $\begin{array}{l}\text { Complicaciones inducidas } \\
\text { por la USEB-ATBT }\end{array}$ \\
\hline $\begin{array}{l}\text { Nakajima et al. [9], 2008, } \\
\text { J Thorac Oncol, Japón }\end{array}$ & $\begin{array}{l}\text { Broncoscopía convencional } \\
\text { sin información diagnóstica } \\
\text { en } 74 \% \text { de los pacientes }\end{array}$ & $63(37-86)$ & 83 & Disponible & $22 \mathrm{G}$ & NR & NR & $\begin{array}{l}\text { Media: } 30 \text {, } \\
\text { (intervalo: } \\
\text { 10-70) }\end{array}$ & $\begin{array}{l}\text { Verificación quirúrgico- } \\
\text { patológica }\end{array}$ & Ninguna \\
\hline $\begin{array}{l}\text { Tournoy et al. [14], 2009, } \\
\text { Lung Cancer, } \\
\text { Bélgica }\end{array}$ & $\begin{array}{l}\text { Broncoscopía convencional } \\
\text { sin información diagnóstica } \\
\text { en } 82 \% \text { de los pacientes }\end{array}$ & $65(43-82)$ & 60 & Disponible & $22 \mathrm{G}$ & NR & $\begin{array}{l}\text { Media: } 21, \\
\text { (intervalo: } \\
\text { 10-60) }\end{array}$ & $\begin{array}{l}\text { Media: } 25, \\
\text { (intervalo: } \\
\text { 10-70) }\end{array}$ & $\begin{array}{l}\text { Verificación quirúrgico- } \\
\text { patológica } \\
\text { Biopsia guiada por TC }\end{array}$ & $\begin{array}{l}\text { Intolerancia del paciente, } \\
\text { con abandono del } \\
\text { procedimiento } \\
n=2(3.3 \%) \\
\text { Fibrilación auricular } \\
\text { autolimitante } \\
n=1(1.7 \%)\end{array}$ \\
\hline $\begin{array}{l}\text { Eckardt et al. } \\
\text { [15], 2010, } \\
\text { World J Surg, Dinamarca }\end{array}$ & $\begin{array}{l}\text { Broncoscopía convencional } \\
\text { sin información diagnóstica } \\
\text { en todos los pacientes } \\
(100 \%)\end{array}$ & $67(29-86)$ & 56 & $\begin{array}{l}\text { No } \\
\text { disponible }\end{array}$ & $22 \mathrm{G}$ & 2 & NR & NR & $\begin{array}{l}\text { Verificación quirúrgico- } \\
\text { patológica } \\
\text { Biopsia guiada por TC } \\
\text { Broncoscopía } \\
\text { Seguimiento clínico }\end{array}$ & Ninguna \\
\hline $\begin{array}{l}\text { Khan et al. } \\
{[16], 2012^{\mathrm{a},}} \\
\text { Thorax, RU }\end{array}$ & $\begin{array}{l}\text { Broncoscopía convencional } \\
\text { sin información diagnóstica } \\
\text { en todos los pacientes } \\
(100 \%)\end{array}$ & 68 (NR) & 47 & NR & NR & $N R$ & NR & NR & $\begin{array}{l}\text { Verificación quirúrgico- } \\
\text { patológica } \\
\text { Biopsia guiada por TC } \\
\text { Seguimiento por TC } \\
\text { exploratoria }\end{array}$ & NR \\
\hline $\begin{array}{l}\text { Bhatti et al. } \\
\text { [17], 2013, Journal of } \\
\text { Bronchology Interv } \\
\text { Pulmonol, EE. UU. }\end{array}$ & NR & 69 (NR) & 50 & NR & $22 \mathrm{G}$ & NR & $\begin{array}{l}\text { Media: 56, } \\
\text { (DE 23) }\end{array}$ & $\begin{array}{l}\text { Media: } 53 \text {, } \\
\text { (DE 24) }\end{array}$ & $\begin{array}{l}\text { Verificación quirúrgico- } \\
\text { patológica } \\
\text { Biopsia guiada por TC }\end{array}$ & $\begin{array}{l}\text { Hemorragia moderada } \\
n=3(9.4 \%) \\
\text { Taquicardia } \\
n=1(3.1 \%)\end{array}$ \\
\hline $\begin{array}{l}\text { Verma et al. } \\
\text { [18], 2013, } \\
\text { Yonsei Med J, } \\
\text { Corea del Sur }\end{array}$ & $\begin{array}{l}\text { Broncoscopía convencional } \\
\text { sin información diagnóstica } \\
\text { en } 40 \% \text { de los pacientes }\end{array}$ & $63(40-81)$ & 68 & $\begin{array}{l}\text { No } \\
\text { disponible }\end{array}$ & $22 \mathrm{G}$ & 2 & NR & $\begin{array}{l}\text { Media } \\
\text { (eje menor): } \\
\text { 27.5, (intervalo: } \\
\text { 8-82) }\end{array}$ & $\begin{array}{l}\text { Verificación quirúrgico- } \\
\text { patológica } \\
\text { Biopsia guiada por TC } \\
\text { Seguimiento clínico ( }>6 \text { meses) }\end{array}$ & $\begin{array}{l}\text { Neumotórax } \\
\mathrm{n}=1(2.7 \%) \\
\text { Hemorragia moderada } \\
\text { autolimitante } \\
\mathrm{n}=1(2.7 \%)\end{array}$ \\
\hline $\begin{array}{l}\text { Yang, et al. } \\
\text { [19], 2013, } \\
\text { Zhonghua Jie He He Hu } \\
\text { Xi Za Zhi, China }\end{array}$ & NR & NR & NR & $\begin{array}{l}\text { No } \\
\text { disponible }\end{array}$ & $22 \mathrm{G}$ & NR & NR & NR & $N R$ & $\begin{array}{l}\text { Hemorragia moderada } \\
\mathrm{n}=1(1.3 \%)\end{array}$ \\
\hline $\begin{array}{l}\text { Zhao et al. } \\
\text { [20], 2013, } \\
\text { Chin Med J, China }\end{array}$ & $\begin{array}{l}\text { Broncoscopía convencional } \\
\text { sin información diagnóstica } \\
\text { en todos los pacientes } \\
(100 \%)\end{array}$ & $56(33-78)$ & 64 & Disponible & $22 \mathrm{G}$ & $3-6$ & NR & $\begin{array}{l}\text { Media } \\
\text { (eje menor): } \\
\text { 34, (intervalo: } \\
20-100 \text { ) }\end{array}$ & $\begin{array}{l}\text { Verificación quirúrgico- } \\
\text { patológica }\end{array}$ & Ninguna \\
\hline $\begin{array}{l}\text { Evison et al. } \\
\text { [21], 2013, } \\
\text { Lung Cancer, RU }\end{array}$ & $\begin{array}{l}\text { Broncoscopía convencional } \\
\text { sin información diagnóstica } \\
\text { en 33\% de los pacientes }\end{array}$ & NR & NR & NR & NR & NR & NR & NR & NR & NR \\
\hline $\begin{array}{l}\text { Argento et al. [22], 2016, } \\
\text { Respir Med, EE. UU. }\end{array}$ & NR & 66 (44-91) & 31 & $\begin{array}{l}\text { No } \\
\text { disponible }\end{array}$ & $22 \mathrm{G}$ & 3 & NR & $\begin{array}{l}\text { Media: } 25.6, \\
\text { (intervalo: } \\
13.2-61.6 \text { ) }\end{array}$ & $\begin{array}{l}\text { Verificación quirúrgico- } \\
\text { patológica } \\
\text { Biopsia guiada por TC } \\
\text { Seguimiento por TC } \\
\text { exploratoria } \\
\text { Biopsia transbronquial AAF-USE }\end{array}$ & Ninguna \\
\hline $\begin{array}{l}\text { Chen et al. } \\
\text { [23], 2017 } \\
\text { Chin Med J (Engl), China }\end{array}$ & $N R$ & $64(19-83)$ & 68 & NR & $\begin{array}{l}21 \mathrm{G} \\
22 \mathrm{G}\end{array}$ & $N R$ & NR & $N R$ & $\begin{array}{l}\text { Verificación quirúrgico- } \\
\text { patológica } \\
\text { Biopsia guiada por TC } \\
\text { Seguimiento por TC } \\
\text { exploratoria } \\
\text { Biopsia transbronquial }\end{array}$ & Ninguna \\
\hline $\begin{array}{l}\text { Almeida et al. } \\
\text { [24], 2018, } \\
\text { J Bronchology Interv } \\
\text { Pulmonol, EE. UU. }\end{array}$ & $\begin{array}{l}\text { Broncoscopía convencional } \\
\text { sin información diagnóstica } \\
\text { en todos los pacientes } \\
(100 \%)\end{array}$ & NR & NR & Disponible & $22 \mathrm{G}$ & $\geq 5$ & NR & $\begin{array}{l}\text { Media: } 43, \\
\text { (intervalo: } \\
\text { 9-131) }\end{array}$ & $\begin{array}{l}\text { Diagnóstico por cualquier otra } \\
\text { técnica } \\
\text { Seguimiento clínico (>3 meses) }\end{array}$ & $\begin{array}{l}\text { Menor (no especificada) } \\
n=4(3.7 \%) \\
\text { Sangrado grave } \\
n=1(0.9 \%) \\
\text { Neumotórax } \\
n=1(0.9 \%)\end{array}$ \\
\hline $\begin{array}{l}\text { Guarize et al. } \\
\text { [25], 2018 } \\
\text { Can Resp J, Canadá }\end{array}$ & NR & $65(20-92)^{c}$ & 63 & Disponible & $22 \mathrm{G}$ & NR & NR & NR & NR & NR \\
\hline $\begin{array}{l}\text { Chaiyakul et al. } \\
\text { [26], 2018, } \\
\text { J Med Assoc Thailand, } \\
\text { Tailandia }\end{array}$ & $\begin{array}{l}\text { Broncoscopía convencional } \\
\text { sin información diagnóstica } \\
\text { en todos los pacientes } \\
(100 \%)\end{array}$ & $59(19-87)$ & 75 & Disponible & $22 \mathrm{G}$ & Media: 5 & $\begin{array}{l}\text { Media: } 45 \text {, } \\
\text { (intervalo: } \\
\text { 20-60) }\end{array}$ & $\begin{array}{l}\text { Media: } 49 \text {, } \\
\text { (intervalo } \\
\text { 22-109) }\end{array}$ & $\begin{array}{l}\text { Verificación quirúrgico- } \\
\text { patológica } \\
\text { Confirmación microbiológica } \\
\text { Biopsia transbronquial } \\
\text { Biopsia guiada por TC } \\
\text { Seguimiento clínico (>3 meses) }\end{array}$ & $\begin{array}{l}\text { Hemorragia menor } \\
n=12(6.9 \%) \\
\text { Desaturación } \\
n=14(8.0 \%)\end{array}$ \\
\hline
\end{tabular}

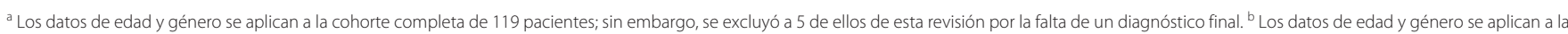
cohorte completa de 308 pacientes; sin embargo, sólo 82 de ellos tuvieron un tumor intrapulmonar. ‘ Los datos de edad y género se aplican a la cohorte completa de 72 pacientes; sin embargo, se excluyó a 6 de ellos de esta revisión por la falta de un diagnóstico final. “ Los datos de edad y género se aplican a la cohorte completa de 1,891 pacientes; sin embargo, sólo 290 de ellos tuvieron un tumor intrapulmonar. NR, no se reportó; USEB-ATBT, ultrasonido endobronquial con biopsia transbronquial por aspiración con aguja; TC, tomografía computarizada; ROSE, evaluación citológica rápida in situ. 
Tabla 3. Rendimiento y sensibilidad de USEB-ATBT para el diagnóstico de lesiones intrapulmonares de localización central

\begin{tabular}{|c|c|c|c|c|c|c|c|}
\hline $\begin{array}{l}\text { Estudio } \\
\text { [Ref.], año }\end{array}$ & $\begin{array}{l}\text { Total de } \\
\text { USEB } \\
\text { realizados, } \\
n\end{array}$ & $\begin{array}{l}\text { Total con } \\
\text { cualquier } \\
\text { malignidad, } \\
n(\%)\end{array}$ & $\begin{array}{l}\text { Muestra tisular } \\
\text { adecuada } \\
\text { mediante USEB- } \\
\text { ATBT, } n(\%)\end{array}$ & $\begin{array}{l}\text { Diagnóstico } \\
\text { correcto por } \\
\text { USEB-ATBT, } n\end{array}$ & $\begin{array}{l}\text { Diagnóstico } \\
\text { correcto de } \\
\text { cualquier } \\
\text { malignidad por } \\
\text { USEB-ATBT, } n\end{array}$ & $\begin{array}{l}\text { Rendimiento para } \\
\text { un diagnóstico } \\
\text { correcto }^{a} \text { (IC 95\%) }\end{array}$ & $\begin{array}{l}\text { Sensibilidad para } \\
\text { malignidad } \\
\text { (IC 95\%) }\end{array}$ \\
\hline $\begin{array}{l}\text { Nakajima et al. } \\
{[9], 2008}\end{array}$ & 35 & $34(97)$ & $35(100)$ & 33 & 32 & $0.94(0.80-0.99)$ & $0.94(0.79-0.99)$ \\
\hline $\begin{array}{l}\text { Tournoy et al. } \\
{[14], 2009}\end{array}$ & 60 & $58(97)$ & $46(77)$ & 46 & 46 & $0.77(0.64-0.86)$ & $0.79(0.67-0.88)$ \\
\hline $\begin{array}{l}\text { Eckardt et al. } \\
{[15], 2010}\end{array}$ & 82 & $51(62 \%)$ & 79 (96) & 59 & 48 & $0.72(0.61-0.81)$ & $0.94(0.83-0.98)$ \\
\hline $\begin{array}{l}\text { Khan et al. } \\
{[16], 2012} \\
\end{array}$ & 114 & $111(95)$ & $113(99)$ & 110 & 108 & $0.96(0.91-0.99)$ & 0.97 (0.92-0.99) \\
\hline $\begin{array}{l}\text { Bhatti et al. } \\
{[17], 2013}\end{array}$ & 32 & $32(100)$ & NR & 30 & 30 & $0.94(0.78-0.98)$ & $0.94(0.78-0.98)$ \\
\hline $\begin{array}{l}\text { Verma et al. } \\
{[18], 2013}\end{array}$ & 37 & $33(89)$ & NR & 32 & 32 & $0.86(0.71-0.94)$ & $0.97(0.81-1.00)$ \\
\hline $\begin{array}{l}\text { Yang et al. } \\
{[19], 2013}\end{array}$ & 78 & $65(83)$ & NR & 75 & 62 & $0.96(0.89-0.99)$ & $0.95(0.87-0.99)$ \\
\hline $\begin{array}{l}\text { Zhao et al. } \\
{[20], 2013}\end{array}$ & 66 & $63(95)$ & $66(100)$ & 59 & 59 & $0.89(0.79-0.95)$ & $0.94(0.84-0.98)$ \\
\hline $\begin{array}{l}\text { Evison et al. } \\
{[21], 2013}\end{array}$ & 49 & $47(95)$ & NR & NR & 38 & - & $0.81(0.67-0.90)$ \\
\hline $\begin{array}{l}\text { Argento et al. } \\
{[22], 2016}\end{array}$ & 32 & $30(94)$ & NR & 27 & 26 & $0.84(0.68-0.93)$ & 0.87 (0.69-0.95) \\
\hline $\begin{array}{l}\text { Chen et al. } \\
\text { [23], } 2017\end{array}$ & 66 & $56(85)$ & NR & 56 & 48 & $0.85(0.74-0.92)$ & $0.86(0.74-0.93)$ \\
\hline $\begin{array}{l}\text { Almeida et al. } \\
{[24], 2018}\end{array}$ & 108 & $93(86 \%)$ & NR & 94 & 88 & $0.87(0.79-0.92)$ & 0.95 (0.88-0.98) \\
\hline $\begin{array}{l}\text { Guarize et al. } \\
{[25], 2018}\end{array}$ & 290 & NR & NR & 266 & 241 & $0.92(0.88-0.94)$ & - \\
\hline $\begin{array}{l}\text { Chaiyakul et al. } \\
{[26], 2018}\end{array}$ & 175 & 147 (84\%) & NR & 158 & 135 & $0.90(0.85-0.94)$ & $0.92(0.86-0.95)$ \\
\hline
\end{tabular}

a El rendimiento se calculó como el número de pacientes para quienes la USEB-ATBT Ilevó a un diagnóstico tisular correcto (maligno o no-maligno) dividido por el número total de pacientes en quienes se practicó el USEB con el fin de diagnosticar un tumor intrapulmonar. b La sensibilidad se calculó como el número de pacientes para quienes USEB-ATBT Ilevó al diagnóstico tisular correcto de una neoplasia, dividido por el número de pacientes en quienes el tumor intrapulmonar bajo estudio resultó ser maligno. NR, no se reportó; USEB, ultrasonido endobronquial; USEB-ATBT, ultrasonido endobronquial con biopsia transbronquial por aspiración con aguja.

0.97 entre los estudios incluidos; un estudio no ofreció información suficiente para calcular la sensibilidad. La sensibilidad media después del metaanálisis fue 0.91 (IC 95\% 0.88-0.94; Fig. 3).

\section{Complicaciones}

En 5 estudios (281 pacientes) no hubo complicaciones debidas al USEB-BTBA, y en 3 estudios (453 pacientes) esta información no se incluyó. En los 6 estudios restantes (490 pacientes) se reportó un total de 42 complicaciones: hemorragia grave $(n=1)$, hemorragia moderada/autolimitante $(\mathrm{n}=17)$, fibrilación auricular $(\mathrm{n}$ $=1)$, taquicardia $(\mathrm{n}=1)$, intolerancia al procedimiento $(\mathrm{n}=2)$, neumotórax $(\mathrm{n}=2)$, desaturación $(\mathrm{n}=14)$ y una complicación menor que no se especificó $(n=4)$. En total, entre los estudios que incluyeron esta información, la tasa de complicaciones fue 5.4\% (42/721), aunque muchas de éstas pueden considerarse menores.

\section{Discusión}

En esta revisión sistemática encontramos que el USEB-BTBA tiene un alto rendimiento y buena sensibilidad para diagnosticar tumores pulmonares de localización central. Los hallazgos de este estudio tienen relevancia clínica, puesto que la adquisición de muestras de tejido de tumores con localización central en pacientes sin anormalidades endobronquiales es un serio problema clínico. El presente análisis parece implicar que, a condición de que el tumor esté ubicado de forma adyacente a las vías respiratorias principales, puede obtenerse un diagnóstico mediante USEBBTBA en aproximadamente 9 de 10 pacientes, con bajo riesgo de complicaciones.

Deben discutirse algunas limitaciones con respecto a los estudios en consideración. Todos los estudios incluidos en esta revisión sistemática presentaron un riesgo elevado de sesgo o problemas de aplicabilidad cuando se evaluaron mediante QUADAS-2 [12]. 


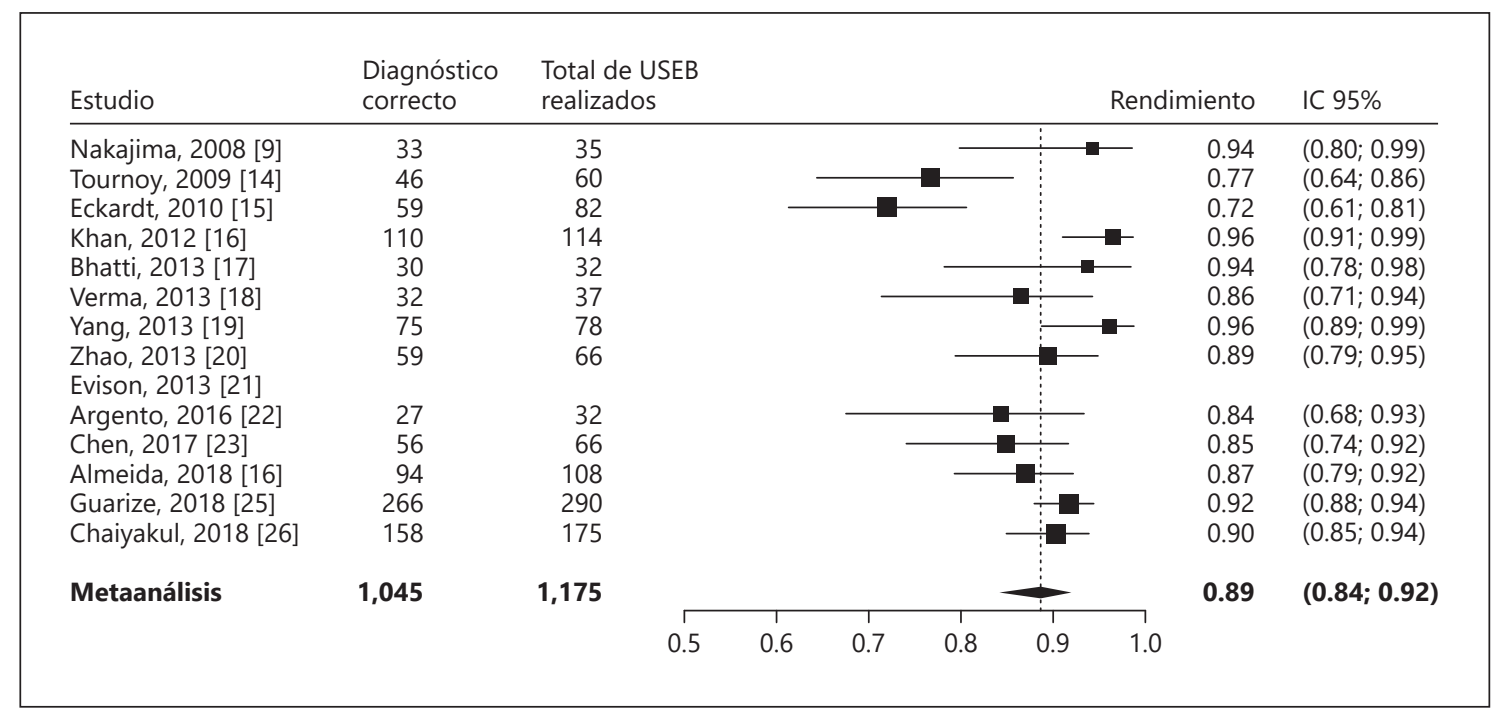

Fig. 2. Rendimiento de USEB-BTBA para diagnosticar lesiones intrapulmonares de localización central. USEB, ultrasonido endobronquial.

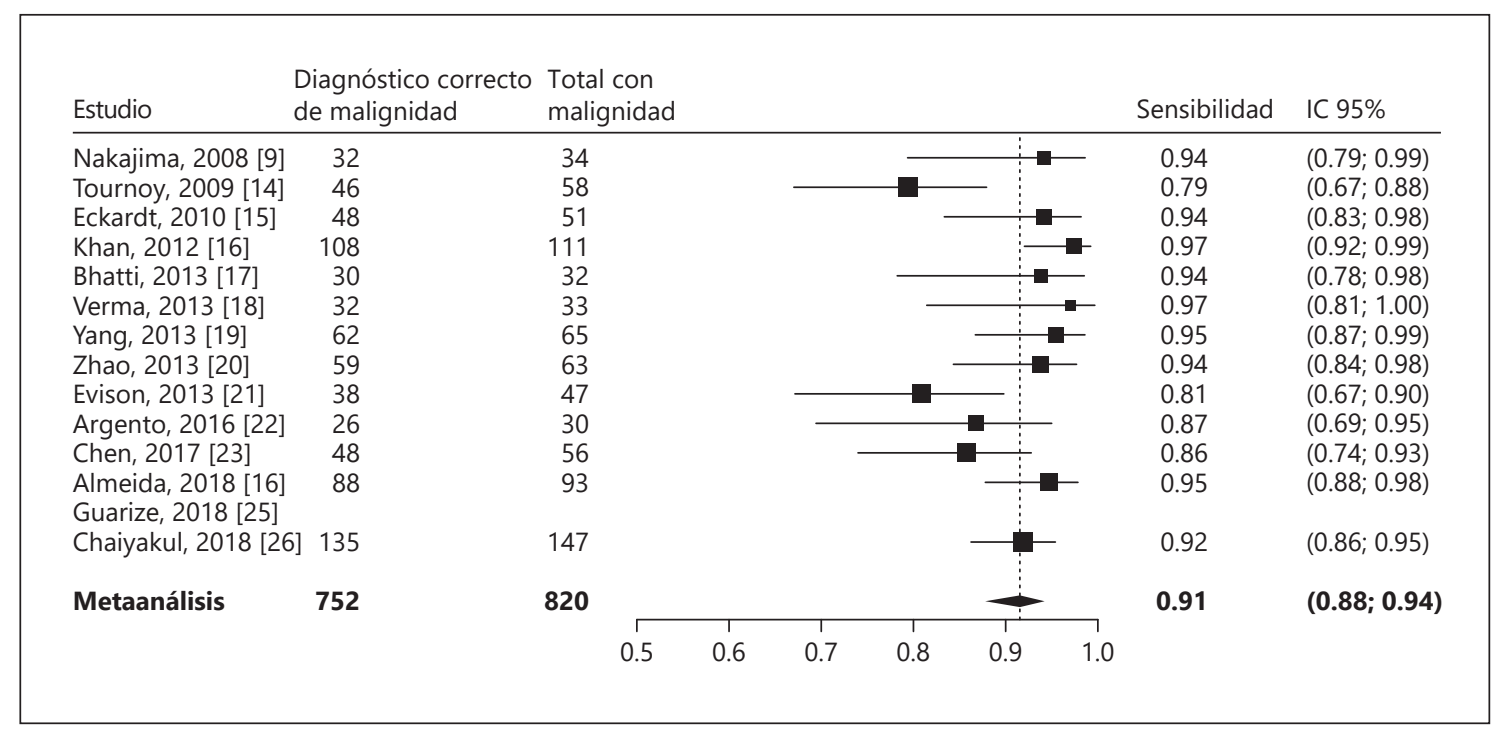

Fig. 3. Sensibilidad de USEB-BTBA para diagnosticar lesiones intrapulmonares de localización central.

Especialmente, el hecho de que casi no se han emprendido estudios prospectivos en el tema es sorprendente. Debido a esto, el rendimiento y la sensibilidad podrían estar sobreestimados. Adicionalmente, en los estudios incluidos se utilizaron varias definiciones diferentes de tumor pulmonar de localización central, desde el tercio interno (lineamientos del Colegio Estadounidense de Especialistas en las Vías Respiratorias) hasta los dos tercios internos (lineamientos de la Sociedad Europea de Cirugía Torácica y la Red General Nacional del Cáncer) [7, 27] del hemitórax.

Variaciones en la experiencia del operador, en el tamaño de la lesión, en su localización con respecto a las vías respiratorias principales y la disponibilidad de ROSE son factores clave que pueden afectar el desempeño del USEB-BTBA [28]. Tal heterogeneidad podría conducir a una variación significativa en el rendimiento y la sensibilidad en diversos entornos clínicos, pero el número limitado de estudios elegibles y la información incompleta en algunos de ellos no nos permitieron realizar análisis de sensibilidad. Sin embargo, las estimaciones promedio del rendimiento y la sensibilidad fueron relativamente constantes entre los estudios individuales, lo que sugiere que el USEB-BTBA puede ser útil en diferentes entornos clínicos.

Siete estudios excluyeron explícitamente o no encontraron pacientes con anormalidades endobronquiales, y 3 estudios explícitamente informaron haber incluido varios pacientes con tales anormalidades. Entre estos 10 estudios, solamente 27 de 660 pacientes (4\%) mostraron anormalidades endobronquiales. Por lo tanto, es poco probable que la presencia de lesiones endobronquiales haya sobreestimado el rendimiento y la sensibilidad del 
USEB-BTBA en el diagnóstico de tumores pulmonares con localización central en nuestra revisión.

Encontramos una alta proporción de pacientes con lesiones malignas en los estudios incluidos. Esto podría, de nuevo, estar relacionado con la naturaleza retrospectiva de la mayoría de los estudios; algunos podrían haber incluido solamente pacientes selectos, con alta probabilidad de malignidad. Es probable que la prevalencia de malignidad sea baja en la práctica.

Solamente ocurrieron complicaciones en $5.8 \%$ de los pacientes, con sólo 2 eventos adversos serios (una hemorragia grave que requirió intervención y un neumotórax). Estos números son comparables con los reportados en estudios previos sobre complicaciones relacionadas con el USEB-BTBA al obtener muestras de nódulos y masas en el mediastino [29]. La complicación más común fue la hemorragia autolimitante, y solamente 2 pacientes presentaron neumotórax debido al USEB-BTBA, lo que sugiere que los rayos $\mathrm{X}$ torácicos de rutina después del USEB-BTBA en tumores intrapulmonares podrían no estar indicados.

El USEB-BTBA es un procedimiento económico para la estadificación del cáncer pulmonar, que puede realizarse en pacientes ambulatorios bajo sedación moderada [30]. Adicionalmente, tiene la ventaja de que puede combinar el diagnóstico de tumores pulmonares y la estadificación loco-regional del mediastino y el hilio en un solo procedimiento. Los resultados de la endosonografía dependen fuertemente de la habilidad del operador, y la técnica debe aprenderse y realizarse de manera sistemática [4, 28]. Se requiere profundizar el aprendizaje y poner en marcha programas de certificación en endosonografía, como el «Programa Integral de Capacitación ERS» para capacitar a doctores calificados para realizar USEB de manera independiente y competente [31]. Además de la evaluación de nódulos, el diagnóstico de tumores intrapulmonares debe formar parte de los programas de capacitación.

Un número sustancial de estudios han evaluado el desempeño del USEB-BTBA en el diagnóstico de tumores mediastinales y en la estadificación de nódulos en el mediastino en pacientes con cáncer pulmonar [32], y esta aplicación se recomienda ahora en la mayoría de los lineamientos clínicos [7,8]. Sin embargo, el número de evaluaciones del desempeño del USEB-BTBA para el diagnóstico de tumores intrapulmonares es limitado, y casi todas son retrospectivas [6]. Con base en nuestra experiencia, creemos que debe considerarse al USEB-BTBA para pacientes con una broncoscopía previa sin información diagnóstica y que presentan un tumor intrapulmonar de localización adyacente o próxima a las vías respiratorias mayores, especialmente cuando las lesiones endobronquiales o metástasis nodulares estén ausentes. Se aconseja emprender estudios futuros, con definiciones claras de tumor pulmonar de localización central, para confirmar los presentes hallazgos. La definición del tumor posicionado en el tercio interno del hemitórax al trazar líneas concéntricas desde la línea media puede ser la más adecuada [33].

A pesar del origen parenquimatoso de la lesión, el USEB lineal parece ser más útil que el USEB radial para el análisis de tumores pulmonares de localización central sin anormalidades endobron- quiales. El USEB radial puede emplearse para detectar lesiones pulmonares, siempre y cuando una vía respiratoria alcance la lesión; sin embargo, en estos casos no es posible una aspiración controlada en tiempo real [34-36].

De igual manera, la BTBA convencional - sin la guía del USEB puede utilizarse para el análisis de tumores pulmonares primarios [2]. La aguja puede colocarse en la carina traqueal ampliada o insertarse en una ubicación específica en las vías respiratorias con base en los hallazgos de la exploración por TC. El rendimiento diagnóstico de la BTBA convencional depende del tamaño y la localización del tumor pulmonar, y se ha reportado un rendimiento diagnóstico de 56\% [2]. Hasta la fecha no se ha emprendido un estudio comparativo entre BTBA guiada por USEB y la BTBA convencional.

Un metaanálisis reciente de nuestro grupo de investigación informó de un alto rendimiento y sensibilidad del USE-(B)-AAF para el diagnóstico de tumores en casos cuando la masa pulmonar se localiza de manera adyacente al esófago [37]. Utilizando USE-(B)AAF, pueden alcanzarse nódulos paraesofágicos inferiores y ubicados a la izquierda [10]. Por tanto, la técnica es complementaria al USEB-BTBA, que permite acceder a estructuras cercanas a las vías respiratorias mayores en ambos lados [38]. Cada vez se aplica más un abordaje combinado de USEB-BTBA y USE-(B)-AAF en la práctica clínica $[3,8]$. Dicho abordaje podría ser útil también en el diagnóstico de tumores intrapulmonares con localización central [39]. Un procedimiento combinado de USEB y USE utilizando sólo el sensor del USEB para el diagnóstico tanto de nódulos como de tumores es una estrategia diagnóstica elegante y mínimamente invasiva.

En conclusión, la presente revisión sistemática y metaanálisis implica que el USEB-BTBA es un procedimiento seguro, con alto rendimiento y sensibilidad para diagnosticar tumores pulmonares de localización central. Sin embargo, debe tenerse cuidado al extrapolar estos resultados en la práctica rutinaria real, debido a la ausencia de estudios de alta calidad. En el futuro, se requieren estudios prospectivos para evaluar si estos hallazgos son reproducibles y para refinar aún más los criterios para recomendar el USEB-BTBA en este entorno.

\section{Declaración de conflictos de interés}

El Dr. Jouke T. Annema recibió apoyo material y financiero para recibir cursos educacionales de USEB por parte de Hitachi, Pentax y COOK; honorarios por conferencias de Pentax y un subsidio a la investigación de COOK Medical. El Dr. Peter I. Bonta reporta subsidios y otros apoyos de Boston Scientific, otro de COOK, otro de Nuvaira, otro de Pentax, apoyo no-financiero de Olympus y subsidios de St Jude Medical. Otros autores: ninguno.

\section{Fuente de financiamiento}

Esta investigación no recibió ningún subsidio específico por parte de organismos de financiamiento en los sectores público, comercial o sin fines de lucro.
44

Kompass Neumol 2020;2:37-45 DOI: $10.1159 / 000506581$ 


\section{Información sobre licencias}

Jolanda C. Kuijvenhoven, Fausto Leoncini, Laurence C. Crombag, René Spijker, Peter I. Bonta, Daniël A. Korevaar, Jouke T. Annema: Ultrasonido endobronquial para el diagnóstico de tumores pulmonares de localización central: Revisión sistemática y metaanálisis [publicado en línea antes de su impresión, 2019 Nov 15]. Respiration. 2019; 1-10 (https:// doi/10.1159/000500363). ${ }^{\circ}$ El (los) autor(es) 2019 Publicado por S. Karger AG, Basilea, autorizado con arreglo a CC BY 4.0 (https://creativecommons. org/licenses/by/4.0/deed.es).

\section{Referencias}

1 Global Burden of Disease Cancer C, Fitzmaurice C, Allen C, Barber RM, et al.: Global, Regional, and National Cancer Incidence, Mortality, Years of Life Lost, Years Lived With Disability, and Disability-Adjusted Life-years for 32 Cancer Groups, 1990 to 2015: A Systematic Analysis for the Global Burden of Disease Study. JAMA Oncol. 2017;3(4):524-548.

2 Rivera MP, Mehta AC, Wahidi MM: Establishing the diagnosis of lung cancer: Diagnosis and management of lung cancer, 3rd ed: American College of Chest Physicians evidence-based clinical practice guidelines. Chest. 2013;143 (5 Suppl):e142S-165S.

3 Boskovic T, Stanic J, Pena-Karan S, et al.: Pneumothorax after transthoracic needle biopsy of lung lesions under CT guidance. J Thorac Dis. 2014;6(Suppl 1):S99-107.

4 Arslan S, Yilmaz A, Bayramgürler B, et al.: CTguided transthoracic fine needle aspiration of pulmonary lesions: accuracy and complications in 294 patients. Med Sci Monit. 2002;8(7): CR493-497.

5 Yung RC: Tissue diagnosis of suspected lung cancer: selecting between bronchoscopy, transthoracic needle aspiration, and resectional biopsy. Respir Care Clin N Am. 2003;9(1):51-76.

6 Vilmann P, Clementsen PF, Colella S, et al.: Combined endobronchial and oesophageal endosonography for the diagnosis and staging of lung cancer. European Society of Gastrointestinal Endoscopy (ESGE) Guideline, in cooperation with the European Respiratory Society (ERS) and the European Society of Thoracic Surgeons (ESTS). Eur Respir J. 2015;46(1):40-60.

7 De Leyn P, Dooms C, Kuzdzal J, et al.: Revised ESTS guidelines for preoperative mediastinal lymph node staging for non-small-cell lung cancer. Eur J Cardiothorac Surg. 2014;45(5): 787-798.

8 Silvestri GA, Gonzalez AV, Jantz MA, et al.: Methods for staging non-small cell lung cancer: Diagnosis and management of lung cancer, 3rd ed: American College of Chest Physicians evidence-based clinical practice guidelines. Chest. 2013;143(5 Suppl):e211S-250S.

9 Nakajima T, Yasufuku K, Fujiwara T, et al.: Endobronchial ultrasound-guided transbronchial needle aspiration for the diagnosis of intrapulmonary lesions. J Thorac Oncol. 2008;3(9):985-988.

10 Colella S, Vilmann P, Konge L, et al.: Endoscopic ultrasound in the diagnosis and staging of lung cancer. Endosc Ultrasound. 2014;3(4):205-212.

11 McInnes MD, Moher D, Thombs BD, et al.; the PRISMA-DTA Group: Preferred Reporting Items for a Systematic Review and Meta-analysis of Diagnostic Test Accuracy Studies: the PRISMADTA Statement. JAMA. 2018;319(4):388-396.

12 Whiting PF, Rutjes AW, Westwood ME, et al.; QUADAS-2 Group: QUADAS-2: a revised tool for the quality assessment of diagnostic accuracy studies. Ann Intern Med. 2011;155(8):529-536.
13 DerSimonian R, Laird N: Meta-analysis in clinical trials. Control Clin Trials. 1986;7(3):177-188.

14 Tournoy KG, Rintoul RC, van Meerbeeck JP, et al.: EBUSTBNA for the diagnosis of central parenchymal lung lesions not visible at routine bronchoscopy. Lung Cancer. 2009;63(1):45-49.

15 Eckardt J, Olsen KE, Licht PB: Endobronchial ultrasound-guided transbronchial needle aspiration of undiagnosed chest tumors. World J Surg. 2010;34(8):1823-1827.

16 Khan SL, Haris M, Diver S, et al.: Can endobronchial ultrasound (EBUS) guided transbronchial needle aspiration (TBNA) be used as a first line investigation in the diagnosis of central lung parenchymal lesion? Thorax 2012;67(Suppl 2):A94.

17 Bhatti HA, Bajwa A, Bhatti JA, et al.: Diagnostic yield of EBUSTBNA for the evaluation of centrally located peribronchial pulmonary lesions. J Bronchology Interv Pulmonol. 2013;20(2): 107-112.

18 Verma A, Jeon K, Koh WJ, et al.: Endobronchial ultrasound guided transbronchial needle aspiration for the diagnosis of central lung parenchymal lesions. Yonsei Med J. 2013;54(3):672-678.

19 Yang HZ, Teng JJ, Zhong RB, et al.: [Endobronchial ultrasound-guided transbronchial needle aspiration in the diagnosis of intrapulmonary lesions]. Zhonghua Jie He He Hu Xi Za Zhi. 2013; 36(1):17-21.

20 Zhao H, Xie Z, Zhou ZL, et al.: Diagnostic value of endobronchial ultrasound guided transbronchial needle aspiration in intrapulmonary lesions. Chin Med J (Engl). 2013;126(22):4312-4315.

21 Evison M, Crosbie P, Martin J, et al: Endobronchial ultrasound in the diagnosis of malignant intra-parenchymal lung lesions - a tertiary centre experience over 2.5 years. Lung Cancer. 2013; 79:S23-24.

22 Argento AC, Puchalski J: Convex probe EBUS for centrally located parenchymal lesions without a bronchus sign. Respir Med. 2016;116:55-58.

23 Chen C, Mu CY, Su MQ, et al.: Endobronchial Ultrasound-guided Transbronchial Needle Aspiration Increases the Yield of Transbronchial Lung Biopsy for the Evaluation of Peribronchial Lesions. Chin Med J (Engl). 2017;130(1):11-14.

24 Almeida FA, Salam S, Mehta AC, et al.: Sampling utility of the convex probe endobronchial ultrasound visible intrapulmonary lesion. J Bronchology Interv Pulmonol. 2018;25(4):290-299.

25 Guarize J, Casiraghi M, Donghi S, et al.: Endobronchial Ultrasound Transbronchial Needle Aspiration in Thoracic Diseases: Much More than Mediastinal Staging. Can Respir J. 2018;2018: 4269798.

26 Chaiyakul S: Endobronchial ultrasound-guided transbronchial needle aspiration for the diagnosis of central intrapulmonary lesions not visible by conventional bronchoscopy. Med Assoc Thai 2018;101(7):939-947.
27 Ettinger DS, Akerley W, Borghaei $\mathrm{H}$, et al.; National comprehensive cancer network: Non-small cell lung cancer, version 2.2013. J Natl Compr Canc Netw. 2013;11(6):645-653.

28 Konge L, Colella S, Vilmann P, Clementsen PF: How to learn and to perform endoscopic ultrasound and endobronchial ultrasound for lung cancer staging: A structured guide and review. Endosc Ultrasound. 2015;4(1):4-9.

29 Vaidya PJ, Munavvar M, Leuppi JD, et al.: Endobronchial ultrasound guided transbronchial needle aspiration: safe as it sounds. Respirology. 2017;22(6):1093-1101.

30 Rintoul RC, Glover MJ, Jackson C, et al.: Cost effectiveness of endosonography versus surgical staging in potentially resectable lung cancer: a health economics analysis of the ASTER trial from a European perspective. Thorax. 2014; 69(7):679-681.

31 Farr A, Clementsen P, Herth F, et al.: Endobronchial ultrasound: launch of an ERS structured training programme. Breathe (Sheff). 2016; 12(3):217-220.

32 Micames CG, McCrory DC, Pavey DA, et al.: Endoscopic ultrasound-guided fine-needle aspiration for non-small cell lung cancer staging: A systematic review and metaanalysis. Chest. 2007; 131(2):539-548.

33 Shin SH, Jeong DY, Lee KS, et al.: Which definition of a central tumour is more predictive of occult mediastinal metastasis in non-small cell lung cancer patients with radiological N0 disease? Eur Respir J. 2019;53(3):1801508.

34 Zaric B, Eberhardt R, Herth F, et al.: Linear and radial endobronchial ultrasound in diagnosis and staging of lung cancer. Expert Rev Med Devices. 2013;10(5):685-695.

35 Steinfort DP, Khor YH, Manser RL, et al.: Radial probe endobronchial ultrasound for the diagnosis of peripheral lung cancer: systematic review and meta-analysis. Eur Respir J. 2011;37(4): 902-910.

36 Ernst A, Anantham D, Eberhardt R, et al.: Diagnosis of mediastinal adenopathy-real-time endobronchial ultrasound guided needle aspiration versus mediastinoscopy. J Thorac Oncol. 2008; 3(6):577-582.

37 Korevaar DA, Colella S, Spijker R, et al.: Esophageal Endosonography for the Diagnosis of Intrapulmonary Tumors: A Systematic Review and Meta-Analysis. Respiration. 2017;93(2):126-137.

38 Vilmann P, Clementsen PF: Combined EUS and EBUS are complementary methods in lung cancer staging: do not forget the esophagus. Endosc Int Open. 2015;3(4):E300-301.

39 Bugalho A, Ferreira D, Eberhardt R, et al.: Diagnostic value of endobronchial and endoscopic ultrasound-guided fine needle aspiration for accessible lung cancer lesions after non-diagnostic conventional techniques: a prospective study. BMC Cancer. 2013;13(1):130. 
Filename: KXN506581

Article-No: 506581, Fig.: 3, Tab.: 3

$<$ Issueid $>2-3</$ Issueid $>$

$532904_{\text {manas ad }}$ J.C.

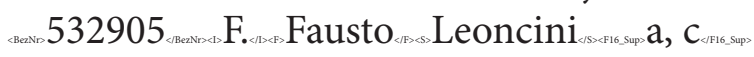

532906

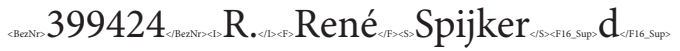

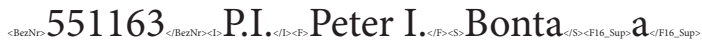

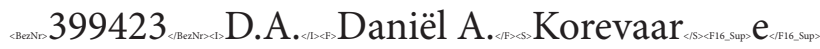

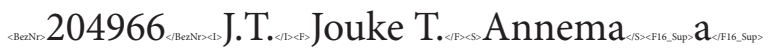

